\title{
Seroprevalencia de anticuerpos contra Trypanosoma cruzi y su asociación con factores de riesgo en menores de 18 años de Veracruz, México
}

\author{
Paz María Salazar, ${ }^{1}$ Gloria Rojas, ${ }^{1}$ Martha Bucio, ${ }^{1}$ Margarita Cabrera, ${ }^{1}$ \\ Guadalupe García, ${ }^{2}$ Adela Ruiz, ${ }^{1}$ Yolanda Guevara ${ }^{1}$ y Roberto Tapia ${ }^{3}$
}

Forma de citar $\begin{aligned} & \text { Salazar PM, Rojas G, Bucio M, Cabrera M, García G, Ruiz A, et al. Seroprevalencia de anticuerpos con- } \\ & \text { tra Trypanosoma cruzi y su asociación con factores de riesgo en menores de } 18 \text { años de Veracruz, Mé- }\end{aligned}$ tra Trypanosoma cruzi y su asociación con factore
xico. Rev Panam Salud Publica. 2007;22(2):75-82.

RESUMEN Objetivos. Determinar la prevalencia de anticuerpos contra Trypanosoma cruzi en menores de 18 años del estado de Veracruz, México, e identificar los factores de riesgo asociados con las características de la vivienda y la presencia y distribución del agente transmisor. Métodos. Estudio epidemiológico transversal en la población menor de 18 años que vivía en 10 de las 11 jurisdicciones sanitarias del estado de Veracruz, México, entre 2000 y 2001. La presencia de anticuerpos contra T. cruzi se determinó mediante una prueba de hemaglutinación indirecta (HAI) y un ensayo inmunoenzimático indirecto tipo ELISA en muestras de suero tomadas en papel de filtro y se confirmaron mediante las pruebas de HAI, ELISA e inmunofluorescencia indirecta (IFI) en muestras de suero. Se aplicó un cuestionario para evaluar las condiciones de la vivienda y se calcularon los indices entomológicos de triatominos intra y peridomiciliarios. Los resultados se evaluaron mediante análisis bifactorial y multifactorial por regresión logística no condicional.

Resultados. De las 150 personas inicialmente reactivas, 14 resultaron positivas (5 mediante la prueba de HAI, ELISA e IFI; 6 por HAI y ELISA y 3 por ELISA e IFI), para una prevalencia general de 0,91\% (IC95\%: 0,85\% a 0,94\%). Los casos positivos residian en cinco jurisdicciones sanitarias y la mayor prevalencia se encontró en Tuxpan: 5,2\% (IC95\%: 1,2\% a 9,0\%). Los factores de riesgo fueron el haber visto chinches dentro de la vivienda y los techos con fisuras. La única especie del agente transmisor capturada fue Triatoma dimidiata. Los indices entomológicos de infestación, colonización e infección natural fueron: 10,9\%, 50,0\% y 9,0\%, respectivamente.

Conclusiones. Se confirmó la transmisión vectorial activa de la infección en el estado de Veracruz, México, con una seroprevalencia general de anticuerpos contra T. cruzi en menores de 18 años de 0,91\%. Se debe seguir atentamente la seroprevalencia en este segmento de la población, como grupo centinela, a fin de evaluar la conveniencia de aplicar medidas más estrictas de control del vector y de vigilancia epidemiológica.

Palabras clave Enfermedad de Chagas, estudios seroepidemiológicos, Trypanosoma cruzi, salud del niño, salud del adolescente, México.

Universidad Nacional Autónoma de México, Facultad de Medicina, Departamento de Microbiología y Parasitología, México, D.F., México. La correspondencia se debe enviar a Paz María Salazar Schettino, Facultad de Medicina, Departamento de Microbiología y Parasitología Edificio " $\mathrm{A}$ ", $2^{\circ}$ piso,
Circuito Escolar, C.P. 04510 México D.F., México. Correo electrónico: pazmar@servidor.unam.mx

2 Departamento de Salud Pública, Facultad de Medicina, Universidad Nacional Autónoma de México, México, D.F., México.
División de Posgrado, Facultad de Medicina, Universidad Nacional Autónoma de México, México, D.F., México. 
En México se desconoce la prevalencia exacta de la enfermedad de Chagas o tripanosomiasis americana, aunque se ha estimado que existen 3-4 millones de personas infectadas por su agente causal, el parásito Trypanosoma cruzi (1). Debido a los pocos estudios epidemiológicos realizados sobre esta enfermedad en México, las autoridades no cuentan con elementos suficientes para considerarla un problema de salud pública. Hasta la fecha no se sabe cuál es la dimensión real de este problema y -aunque se han realizado estudios seroepidemiológicos, clínicos y de transmisores y reservorios- aún no se ha logrado caracterizar su distribución, prevalencia y dinámica de transmisión en el país. La mayoría de estos estudios se han realizado con metodologías y criterios muy diversos, lo que dificulta hacer un análisis válido de la información. Sin embargo, hay un hecho real y aceptado: en México se ha detectado el vector transmisor infectado, existen los reservorios habituales y han enfermado personas, lo que significa que la enfermedad está presente (2-9). La presentación, evolución y repercusión de esta enfermedad depende en gran medida de las características del hospedero.

Como parte del proyecto "Importancia de la enfermedad de Chagas en el estado de Veracruz, México" - realizado con apoyo financiero parcial del Programa Especial para Investigación y Entrenamiento en Enfermedades Tropicales de la Organización Mundial de la Salud (OMS) mediante el proyecto No. 970854, y con la participación del laboratorio de Biología de Parásitos de la Facultad de Medicina de la Universidad Nacional Autónoma de México y de la Secretaría de Salud del estado de Veracruz- se estimó la prevalencia de esta enfermedad en menores de 18 años de edad. Se seleccionó este estado por contar con condiciones socioeconómicas, climáticas y ecológicas apropiadas para la transmisión activa del T. cruzi. Por otra parte, este grupo que abarca toda la edad pediátrica puede servir de centinela de la transmisión activa de este parásito, algo de gran importancia epidemiológica si se toma en cuenta que la inte- rrupción de la transmisión es el mejor indicador de la eficacia de los programas de control. Estudios similares llevados a cabo en América del Sur se han basado en personas de 7 a 14 años (edad escolar) (10-18).

A partir del estudio de Hoffman en 1928 (19) se estableció que la especie de triatomino Conorrhinus dimidiatus (Stal, 1859 y Champion, 1899), actualmente denominada Triatoma dimidiata (Latreille) (20), es la responsable de la transmisión de la enfermedad de Chagas en México (19). Diversos estudios realizados en las décadas siguientes han confirmado la asociación de este triatomino con la transmisión de T. cruzi en todos los estados mexicanos. (20-25). Otras especies importantes en este sentido son Rhodnius prolixus, Triatoma barberi, Triatoma gerstaeckeri, Triatoma longipennis, Triatoma mazzottii, Triatoma mexicana, Triatoma pallidipennis, Triatoma phyllosoma y Triatoma picturata (25).

En el estado de Veracruz, las especies informadas son Triatoma dimidiata (19-24), Triatoma gerstaeckeri (24), Triatoma pallidipennis $(20,22)$, Triatoma rubida (20), Panstrongylus geniculatus (26), Panstrongylus rufotuberculatus $(20,23)$ y Eratyrus cuspidatus $(22,26,27)$.

Para poder elaborar y poner en práctica intervenciones eficaces para el control de la transmisión y establecer un sistema efectivo de vigilancia epidemiológica, particularmente en niños y niñas, es necesario ampliar el conocimiento acerca de las características epidemiológicas y los factores de riesgo de la infección por T. cruzi.

El objetivo principal de esta investigación fue determinar la prevalencia de anticuerpos contra T. cruzi en menores de 18 años del estado de Veracruz, México, e identificar los factores de riesgo asociados con las características de la vivienda y la presencia y distribución del agente transmisor.

\section{MATERIALES Y MÉTODOS}

Se realizó un estudio epidemiológico transversal en la población menor de 18 años que vivía en 10 de las 11 jurisdicciones sanitarias del estado de Veracruz, México, entre marzo de 2000 y marzo de 2001. Al momento de comenzar el estudio, la población de ese estado era de 7059360 habitantes, de los cuales 2951947 (41,8\%) tenían menos de 18 años.

El estado de Veracruz está dividido en 11 jurisdicciones sanitarias. Para calcular el tamaño de la muestra de cada jurisdicción se utilizó un procedimiento estándar (28) a partir de una prevalencia de $15 \%$, un nivel de confianza de $95 \%$ y una precisión de $2 \%$, según otros estudios realizados en México (1-9). El número aproximado de viviendas se estimó dividiendo la población total de cada jurisdicción entre 5, que es el promedio estimado de personas que viven en cada vivienda. La muestra correspondiente a cada jurisdicción se distribuyó proporcionalmente entre sus localidades según su número de habitantes (1-99, 100-499, 500-999 y 1000 o más). Las viviendas se escogieron al azar mediante una tabla de números aleatorios (dos por manzana en cada localidad). Se decidió trabajar con las localidades de menos de 500 habitantes ya que los focos de transmisión de la enfermedad de Chagas están ubicados principalmente en localidades rurales y de poca población. En cada vivienda elegida se estudiaron todas las personas menores de 18 años de edad que hubieran vivido en esa comunidad al menos durante el último año y que nunca hubieran recibido transfusiones sanguíneas.

Para el tamizaje se tomaron muestras de sangre en papel filtro Whatman No. 1 (Whatman Laboratory Division, Maidstone, Reino Unido). La presencia de anticuerpos contra $T$. cruzi se determinó mediante dos pruebas serológicas: una de hemaglutinación indirecta (HAI) y un ensayo inmunoenzimático indirecto tipo ELISA (29-31). Los casos reactivos se confirmaron posteriormente mediante las pruebas de HAI, ELISA e inmunofluorescencia indirecta (IFI) con muestras de suero de sangre periférica obtenida por punción venosa en el brazo.

Las tres pruebas se realizaron con antígenos extraídos de una cepa de T. cruzi aislada en México. El antígeno para la prueba de HAI se preparó conjuntamente con personal del Instituto 
Nacional de Chagas Dr. Mario Fatala Chabén, de Argentina, según la metodología desarrollada en ese instituto. El análisis se realizó en microplacas de 96 pozos con fondo en $\mathrm{U}$ (Costar, Corning Incorporated, Nueva York, Estados Unidos de Amçerica) con diluciones de la muestra de 1:16, 1:32 y 1:64 en solución tampón salina de fosfato (PBS, $\mathrm{pH}$ $7,2)(29)$. Las muestras en papel se consideraron reactivas a partir de un título de 1:16, mientras que las muestras de suero se consideraron positivas a partir de un título de 1:32. La prueba de ELISA se llevó a cabo según la metodología descrita por Voller (30) con un antígeno de recubrimiento previamente caracterizado (31). Las pruebas de IFI se realizaron en el Laboratorio de Inmunoparasitología del Departamento de Microbiología y Parasitología de la Universidad Nacional Autónoma de México, según el método estándar (32) con un antígeno caracterizado en nuestro laboratorio (33).

La validación inicial de las tres pruebas se realizó con paneles de suero proporcionados por el Instituto Nacional de Chagas Dr. Mario Fatala Chabén. Para la prueba de HAI se utilizó un panel de 20 sueros (10 positivos y 10 negativos) y para las de ELISA e IFI se empleó un panel de 50 sueros (25 positivos y 25 negativos). En todos los casos se calcularon la sensibilidad, la especificidad y los valores predictivos positivo y negativo.

Los menores estudiados se consideraron positivos a anticuerpos contra T. cruzi si sus muestras de suero resultaban reactivas al menos en dos de las tres pruebas realizadas.

La información relacionada con las variables biológicas (edad, sexo y presencia de anticuerpos contra T. cruzi), socioculturales (escolaridad y ocupación) y de estilo de vida (presencia de animales, hacinamiento en la vivienda y rociar insecticidas habitualmente) se obtuvieron mediante un cuestionario aplicado directamente al padre o la madre del menor o a los jóvenes mayores de 15 años, si no se encontraba ninguno de los padres. Los datos necesarios para evaluar los factores de riesgo de la vivienda se consignaron según el criterio de los entrevistado-

\section{CUADRO 1. Clasificación del material de construcción de las viviendas según el riesgo de albergar triatominos}

\begin{tabular}{lll}
\hline \multicolumn{1}{c}{ Techo } & \multicolumn{1}{c}{ Muros } & Piso \\
\hline $\begin{array}{l}\text { Viviendas con riesgo } \\
\text { Madera/corteza }\end{array}$ & Adobe & Tierra \\
Carrizo/bambú & Barro & Madera \\
Palma/zacate & Piedras & \\
Tejas & Madera & \\
Láminas de cartón & Carrizo/bambú & \\
& Láminas de cartón & \\
Viviendas sin riesgo & & Mosaicos \\
Cemento/concreto & & Piedras \\
Láminas de asbesto & Cemento & \\
Láminas de zinc & Ladrillos & \\
\hline Fuente: Referencia 34. & Bloques & \\
& &
\end{tabular}

res, previamente entrenados para ello. Los criterios para clasificar el material de construcción de la vivienda según el riesgo de albergar triatominos se muestran en el cuadro 1 (34). La búsqueda de los vectores transmisores se realizó tanto dentro como en los alrededores de la vivienda y se calcularon los índices entomológicos (35).

Se requirió una carta de consentimiento informado firmada por alguno de los padres de los menores participantes en el estudio para recabar la información, evaluar la vivienda y tomar las muestras de sangre.

La descripción estadística se realizó a partir de los porcentajes de las variables cualitativas. Se determinó la frecuencia de los factores de riesgo y de la positividad a anticuerpos contra T. cruzi y se analizó si existían diferencias significativas según los grupos de edad y el sexo mediante la prueba de la ji al cuadrado. Para el análisis de los factores de riesgo se tomaron como controles cuatro casos negativos por cada caso positivo, pareados por sexo y edad ( \pm 2 años). La fuerza de la asociación de los factores de riesgo con la seropositividad (análisis bifactorial) se evaluó mediante la razón de posibilidades (odds ratio, OR) y su significación estadística mediante la prueba de la ji de Mantel y Haenszel y los intervalos de confianza de 95\% (IC95\%). Se empleó un nivel de significación estadística de $P<0,05$.

A fin de precisar las variables asociadas con el riesgo de portar anticuerpos contra T. cruzi, se realizó un análisis de regresión logística no condicional mediante el programa SPSS v. 10. Para ello se emplearon todas las variables que en el análisis bifactorial presentaron un nivel de significación de $P<$ 0,20 (36). Para evaluar la proporción de personas seropositivas atribuibles a cada uno de los factores asociados y poder estimar la proporción de seropositivos que podría haberse evitado si se eliminaran esos factores de riesgo (impacto potencial) se calculó la fracción etiológica en las personas expuestas a cada uno de estos factores.

\section{RESULTADOS}

El estudio de validación con paneles previamente caracterizados dio como resultado que la sensibilidad de la técnica de HAI fue de $100 \%$, la especificidad de $50,0 \%$, el valor predictivo positivo de $66,6 \%$ y el valor predictivo negativo de $100 \%$ (cuadro 2). Para la técnica de ELISA se obtuvo una sensibilidad de $96,0 \%$, especificidad de $100 \%$ y los valores predictivos positivo y negativo fueron de $100 \%$ y $96,1 \%$, respectivamente (cuadro 3). Para la técnica de IFI, la sensibilidad fue de $88,0 \%$, la especificidad de $100 \%$ y los valores predictivos positivo y negativo de $100 \%$ y $89,2 \%$, respectivamente (cuadro 4 ).

En total se estudiaron 1544 menores de 18 años de Veracruz, de ellos 731 $(47,3 \%)$ eran varones y $813(52,7 \%) \mathrm{mu}-$ jeres, con un promedio de edad de 10 años. En el tamizaje realizado con muestras tomadas en papel filtro, 1377 
CUADRO 2. Resultados de la validación de la prueba de hemaglutinación indirecta (HAI) para detectar anticuerpos contra Trypanosoma cruzi en suero

\begin{tabular}{lrrr}
\hline & \multicolumn{3}{c}{ Característica } \\
\cline { 2 - 4 } HAI & 10 & 5 & 15 \\
\cline { 2 - 4 } de las muestras \\
\hline Positivos & 0 & 5 & 5 \\
Negativos & 10 & 10 & 20 \\
$\quad$ Total & & Negativas & Total \\
\hline
\end{tabular}

Sensibilidad $=100 \%$; especificidad $=50,0 \%$; valor predictivo positivo $=66,6 \% ; y$ valor predictivo negativo $=100 \%$.

CUADRO 3. Resultados de la validación de la prueba de ELISA para detectar anticuerpos contra Trypanosoma cruzi en suero

\begin{tabular}{lrrr}
\hline & \multicolumn{3}{c}{ Característica } \\
ELISA & 24 & 0 & 24 \\
\cline { 2 - 4 } & Positivas & Negativas & Total \\
\hline Positivos & 1 & 25 & 26 \\
Negativos & 25 & 25 & 50 \\
$\quad$ Total & & & \\
\hline
\end{tabular}

Sensibilidad $=96,0 \%$; especificidad $=100 \%$; valor predictivo positivo $=100 \% ; y$ valor predictivo negativo $=96,1 \%$.

CUADRO 4. Resultados de la validación de la prueba de inmunofluorescencia indirecta (IFI) para detectar anticuerpos contra Trypanosoma cruzi en suero

\begin{tabular}{lrcc}
\hline & \multicolumn{3}{c}{$\begin{array}{c}\text { Característica } \\
\text { de las muestras }\end{array}$} \\
\cline { 2 - 4 } IFI & Positivas & Negativas & Total \\
\hline Positivos & 22 & 0 & 22 \\
Negativos & 3 & 25 & 28 \\
Total & 25 & 25 & 50 \\
\hline
\end{tabular}

Sensibilidad $=88,0 \%$; especificidad $=100 \%$; valor predictivo positivo $=100 \% ; y$ valor predictivo negativo $=89,2 \%$.

$(89,2 \%)$ resultaron negativas por $\mathrm{HAI}$ y ELISA, mientras que $167(10,8 \%)$ resultaron reactivas: $138(82,6 \%)$ de ellas mediante HAI, $23(13,8 \%)$ mediante ELISA y $6(3,6 \%)$ por ambas pruebas.

Se recibieron muestras de suero para el análisis confirmatorio de 150 personas cuyas muestras resultaron reactivas en el estudio de tamizaje, de ellas 14 resultaron positivas (5 mediante la prueba de HAI, ELISA e IFI; 6 por HAI y ELISA y 3 por ELISA e IFI) (cuadro 5). Según estos resultados, la prevalencia encontrada fue de 0,91\% (IC95\%: 0,85\% a $0,94 \%$ ). La mitad de estos casos eran varones. Solamente se encontró dife-

CUADRO 5. Características de los menores de 18 años de edad positivos a anticuerpos contra Trypanosoma cruzi y los resultados de las pruebas diagnósticas empleadas. Estado de Veracruz, México, marzo de 2001

\begin{tabular}{|c|c|c|c|c|c|c|c|c|}
\hline \multirow[b]{2}{*}{ No. } & \multirow{2}{*}{$\begin{array}{l}\text { Jurisdicción } \\
\text { sanitaria }^{a}\end{array}$} & \multirow[b]{2}{*}{ Sexo } & \multirow{2}{*}{$\begin{array}{l}\text { Edad } \\
\text { (años) }\end{array}$} & \multicolumn{2}{|c|}{$\begin{array}{l}\text { Resultados de las } \\
\text { muestras tomadas } \\
\text { en papel de filtro }\end{array}$} & \multicolumn{3}{|c|}{$\begin{array}{l}\text { Resultados de } \\
\text { las muestras } \\
\text { de suero }\end{array}$} \\
\hline & & & & ELISA $^{b}$ & $\mathrm{HAl}^{\mathrm{C}}$ & ELISA $^{b}$ & $\mathrm{HAl}^{\mathrm{C}}$ & $\mathrm{IFI}^{\mathrm{d}}$ \\
\hline 1 & 1 & $F$ & 10 & - & + & + & + & + \\
\hline 2 & 1 & $\mathrm{~F}$ & 7 & - & + & + & + & - \\
\hline 3 & 1 & $M$ & 17 & - & + & + & + & - \\
\hline 4 & 2 & $\mathrm{~F}$ & 7 & + & - & + & + & - \\
\hline 5 & 2 & $\mathrm{~F}$ & 17 & + & - & + & - & + \\
\hline 6 & 2 & $\mathrm{~F}$ & 12 & + & - & + & + & + \\
\hline 7 & 2 & M & 4 & + & - & + & + & - \\
\hline 8 & 2 & $\mathrm{~F}$ & 10 & + & - & + & + & - \\
\hline 9 & 2 & $M$ & 5 & + & - & + & + & + \\
\hline 10 & 6 & M & 13 & + & - & + & + & - \\
\hline 11 & 6 & M & 9 & - & + & + & + & + \\
\hline 12 & 6 & M & 2 & - & + & + & - & + \\
\hline 13 & 8 & M & 17 & - & + & + & + & + \\
\hline 14 & 10 & $\mathrm{~F}$ & 14 & + & - & + & - & + \\
\hline
\end{tabular}

1: Pánuco $(n=118) ; 2$ : Tuxpan $(n=116) ; 6$ : Córdoba $(n=170) ; 8$ : Veracruz $(n=244) ; 10$ : San Andrés Tuxtla $(n=139)$. b Prueba inmunoenzimática indirecta tipo ELISA.

c Prueba de hemaglutinación indirecta.

d Prueba de inmunofluorescencia indirecta.

rencia estadísticamente significativa $(P=0,01)$ entre niños y niñas del grupo de 10 a 14 años (cuadro 6). La mayor prevalencia se observó en la jurisdicción sanitaria de Tuxpan: 5,2\% (IC95\%: $1,2 \%$ a $9,0 \%$ ), con 6 positivos de 116 encuestados (figura 1).

Según el análisis bifactorial, los factores asociados significativamente con la seropositividad fueron el uso de material de riesgo en el piso de la vivienda $(P=0,02)$, la presencia de fisuras en el piso $(P=0,03)$ y el haber visto chinches dentro de la vivienda $(P=0,01)$. No obstante, para modelar los datos y encontrar los factores que en su conjunto pudieran explicar la seropositividad a anticuerpos contra $T$ cruzi, también se incorporaron al modelo logístico las variables con valores de $P<0,20$ : reconocer las chinches $(P=0,09)$, que los animales durmieran dentro del domicilio $(P=0,09)$, escasa ventilación del dormitorio $(P=0,20)$, presencia de fisuras en el techo $(P=0,10)$, encontrar chinches fuera de la casa $(P=0,09)$ y no rociar insecticidas habitualmente $(P=$ 0,14) (cuadro 7).

El resultado del análisis multifactorial mostró que los factores que se mantuvieron en el modelo por su significación estadística (cuadro 8) fueron el haber visto chinches dentro de la vivienda, con un riesgo de positividad para $T$. cruzi 5,3 veces $(\mathrm{OR}=5,3$; IC95\%: $1,46$ a 19,5$)$ mayor que en las viviendas donde no se habían visto; y las fisuras en el techo, con un riesgo 3,7 veces $(\mathrm{OR}=3,69$; IC95\%: 0,96 a 14,2) mayor de ser seropositivos a anticuerpos contra T. cruzi que los que vivían en viviendas sin fisuras en el techo. Este modelo resultó significativo según la prueba de Hosmer-Lemeshow $(0,935)$.

De las especies transmisoras, Triatoma dimidiata fue la única especie encontrada, tanto dentro de la vivienda como en su entorno. El análisis de los índices entomológicos en las jurisdicciones sanitarias en las que se detectaron menores de 18 años seropositivos a la infección por T. cruzi muestra que los índices de infestación más elevados correspondieron a las jurisdicciones sanitarias de Pánuco (29,0\%) y Tuxpan (20,0\%). En estos lugares se presentó el mayor número de personas seropositivas. El índice de colonización más elevado se encontró en la jurisdicción sanitaria de San Andrés Tuxtla (100\%) y los de infección natural más elevados correspondieron a las jurisdicciones sanitarias de Veracruz (14,3\%) y Pánuco $(13,3 \%)$ (figura 2$)$. 
CUADRO 6. Seroprevalencia de la infección por Trypanosoma cruzi en la población menor de 18 años, según la edad y el sexo. Estado de Veracruz, México, marzo de 2001

\begin{tabular}{|c|c|c|c|c|c|c|c|}
\hline \multirow{2}{*}{$\begin{array}{l}\text { Edad } \\
\text { (años) }\end{array}$} & \multicolumn{2}{|c|}{ Niños y adolescentes } & \multicolumn{2}{|c|}{ Niñas y adolescentes } & \multicolumn{2}{|c|}{ Total } & \multirow[b]{2}{*}{$P^{a}$} \\
\hline & Positivos/total & $\%$ & Positivos/total & $\%$ & Positivos/total & $\%$ & \\
\hline 1 a 4 & $2 / 93$ & 2,15 & $0 / 87$ & 0 & $2 / 180$ & 1,11 & - \\
\hline 5 a 9 & 2/237 & 0,84 & $2 / 244$ & 0,82 & $4 / 481$ & 0,83 & 0,63 \\
\hline 10 a 14 & $1 / 300$ & 0,33 & $4 / 304$ & 1,32 & $5 / 604$ & 0,83 & 0,01 \\
\hline 15 a 18 & $2 / 101$ & 1,98 & $1 / 178$ & 0,56 & $3 / 279$ & 1,08 & 0,8 \\
\hline Total & $7 / 731$ & 1,00 & $7 / 813$ & 0,86 & $14 / 1544$ & 0.91 & 0.8 \\
\hline
\end{tabular}

a Nivel de significación $P<0,05$.

FIGURA 1. Prevalencia (\%) de anticuerpos contra Trypanosoma cruzi en la población menor de 18 años, según la jurisdicción sanitariaa. Estado de Veracruz, México, marzo de 2001

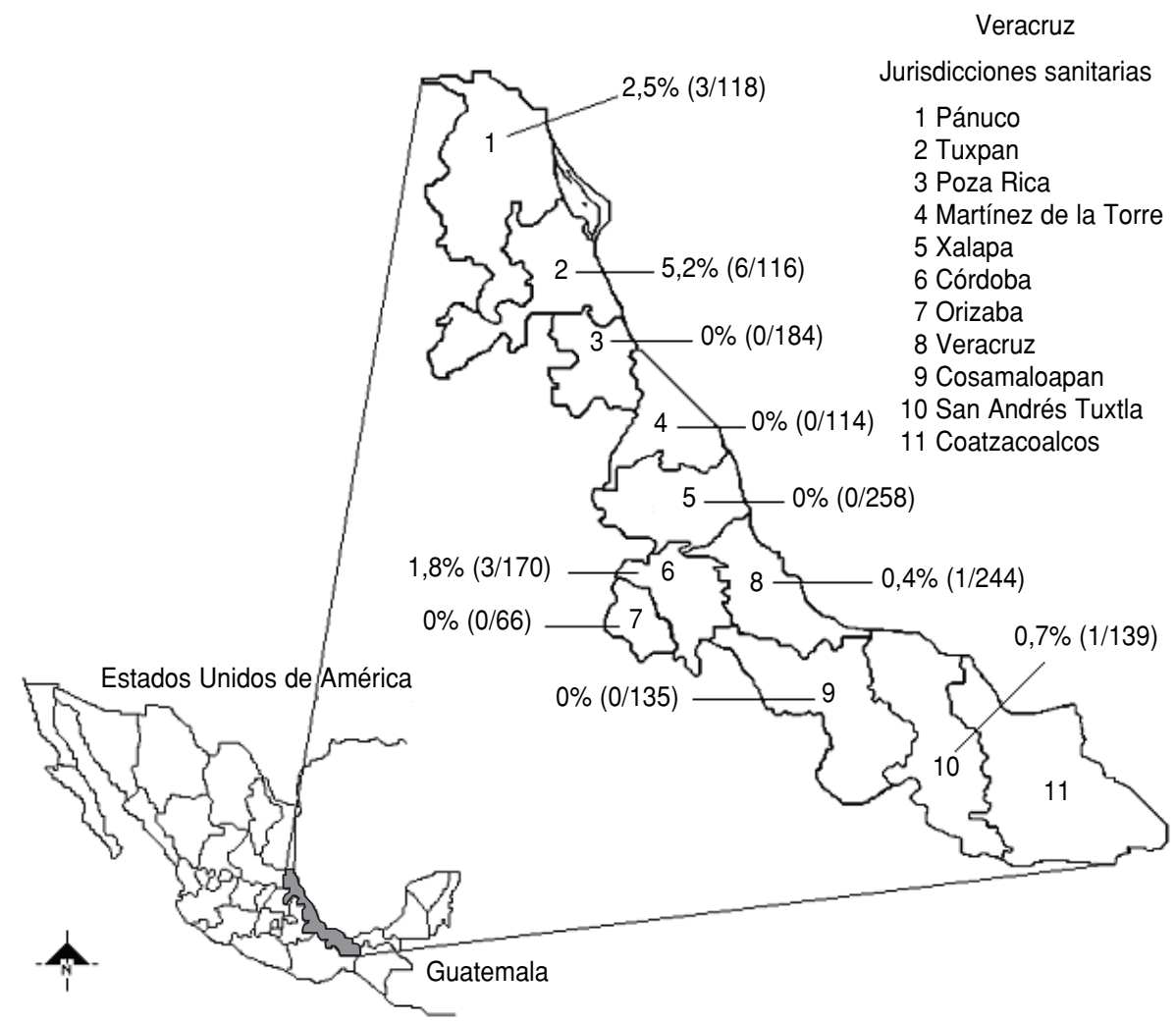

a Entre paréntesis se ofrecen los datos del estudio: el numerador corresponde al número de seropositivos y el denominador al número de personas estudiadas en la jurisdicción sanitaria dada.

\section{DISCUSIÓN}

Según estudios realizados en el estado de Veracruz, la prevalencia de anticuerpos contra T. cruzi varió entre 3\% en población abierta (1), 1\% en donantes de sangre del estado (37) y $4 \%$ en donadores provenientes de todo el país (38). Ningún estudio había investigado hasta el momento la presencia de estos anticuerpos en la población menor de 18 años.
En el presente estudio, la prevalencia en menores de 18 años fue de 0,91\%. La correlación encontrada entre los resultados obtenidos a partir de las muestras fijadas en papel de filtro y de los sueros respectivos es baja, posiblemente debido a artefactos en las determinaciones realizadas con las primeras, por la exposición a condiciones inadecuadas de almacenamiento y transportación (39).
Las pruebas serológicas empleadas en este estudio son las recomendadas por la OMS para la detección de anticuerpos contra T. cruzi (40). Aunque los resultados obtenidos mediante la técnica de HAI con el panel de sueros mostraron una baja especificidad de $50 \%$ - característica de esta técnica-, su valor predictivo negativo fue de $100 \%$. Por esta razón, fue apropiado utilizarla como prueba de tamizaje, ya que redujo el riesgo de perder muestras positivas.

Se encontraron casos positivos en 5 de las 10 jurisdicciones sanitarias estudiadas, con prevalencias de 0,4 a 5,2\% (figura 1). En general, 36\% de esos casos eran menores de 7 años, por lo que el control y la vigilancia deben ser prioridades de las autoridades sanitarias de esas jurisdicciones, especialmente en Tuxpan y Pánuco, donde se encontraron las mayores prevalencias (5,1\% y 2,5\%, respectivamente). El hecho de que los índices entomológicos de infestación en esas jurisdicciones fueran de $30 \%$ y 35\%, respectivamente, y que los índices de colonización se elevaran a $77 \%$ y $81 \%$, respectivamente, indica que el ciclo de vida del transmisor transcurre dentro de la vivienda. El índice de infección natural con T. cruzi fue de 13\% en Pánuco y de $9 \%$ en Tuxpan, similares a los encontrados por Salvatella y colaboradores en 1999 (10). El análisis de los índices entomológicos y de la seroprevalencia permitieron determinar que las zonas de mayor transmisión fueron las jurisdicciones sanitarias del norte del estado (Pánuco y Tuxpan) y confirma que la presencia del vector constituye un elemento importante en la dinámica de transmisión de T. cruzi. 
CUADRO 7. Asociación entre los diferentes factores de riesgo de la enfermedad de Chagas, según el modelo multifactorial. Estado de Veracruz, México, marzo de 2001

\begin{tabular}{|c|c|c|c|c|c|}
\hline Variable & $\begin{array}{l}\text { Positivos } \\
(n=14)\end{array}$ & $\begin{array}{c}\text { Controles } \\
(n=56)\end{array}$ & $\mathrm{OR}^{\mathrm{a}}$ & IC95\% ${ }^{b}$ & $P^{c}$ \\
\hline \multicolumn{6}{|l|}{ Conoce las chinches } \\
\hline Sí & 13 & 39 & 5,67 & \multirow[t]{2}{*}{$0,67-125,2$} & \multirow[t]{2}{*}{0,09} \\
\hline No & 1 & 17 & 1,00 & & \\
\hline \multicolumn{6}{|l|}{ Convive con perros } \\
\hline Sí & 10 & 42 & 0,83 & \multirow[t]{2}{*}{$0,19-3,77$} & \multirow[t]{2}{*}{0,74} \\
\hline No & 4 & 14 & 1,00 & & \\
\hline \multicolumn{6}{|l|}{ Convive con gatos } \\
\hline Sí & 7 & 22 & 1,55 & \multirow[t]{2}{*}{$0,41-5,82$} & \multirow[t]{2}{*}{0,67} \\
\hline No & 7 & 34 & 1,00 & & \\
\hline \multicolumn{6}{|l|}{ Lugar donde duermen los animales } \\
\hline Dentro del domicilio & 2 & 1 & 9,17 & \multirow[t]{2}{*}{$0,57-280,2$} & \multirow[t]{2}{*}{0,09} \\
\hline En los alrededores del domicilio & 12 & 55 & 1,00 & & \\
\hline \multicolumn{6}{|l|}{ Material del techo } \\
\hline Con riesgo & 5 & 18 & 1,17 & \multirow[t]{2}{*}{$0,29-4,63$} & \multirow[t]{2}{*}{0,95} \\
\hline Sin riesgo & 9 & 38 & 1,00 & & \\
\hline \multicolumn{6}{|l|}{ Material de los muros } \\
\hline Con riesgo & 11 & 36 & 2,04 & \multirow[t]{2}{*}{$0,45-10,5$} & \multirow[t]{2}{*}{0,36} \\
\hline Sin riesgo & 3 & 20 & 1,00 & & \\
\hline \multicolumn{6}{|l|}{ Material del piso } \\
\hline Con riesgo & 13 & 31 & 10,48 & \multirow[t]{2}{*}{$1,26-229,2$} & \multirow[t]{2}{*}{0,02} \\
\hline Sin riesgo & 1 & 25 & 1,00 & & \\
\hline \multicolumn{6}{|l|}{ Ventilación del dormitorio } \\
\hline Buena & 2 & 19 & 0,32 & $0,04-1,79$ & 0,20 \\
\hline Deficiente & 12 & 37 & 1,00 & & \\
\hline Iluminación del dormitorio & & & & & \\
\hline Buena & 2 & 17 & 0,38 & $0,05-2,13$ & 0,32 \\
\hline Deficiente & 12 & 39 & 1,00 & & \\
\hline Fisuras en el techo & & & & & \\
\hline Sí & 10 & 24 & 3,33 & $0,82-14,6$ & 0,10 \\
\hline No & 4 & 32 & 1,00 & & \\
\hline Fisuras en los muros & & & & & \\
\hline Sí & 12 & 46 & 1,30 & $0,22-9,9$ & 1,0 \\
\hline No & 2 & 10 & 1,00 & & \\
\hline Fisuras en el piso & & & & & \\
\hline Sí & 11 & 24 & 4,89 & $1,08-25,1$ & 0,03 \\
\hline No & 3 & 32 & 1,00 & & \\
\hline Se vieron chinches dentro de la ca & & & & & \\
\hline Sí & 9 & 15 & 4,92 & $1,23-2,06$ & 0,01 \\
\hline No & 5 & 41 & 1,00 & & \\
\hline Se vieron chinches fuera de la cas & & & & & \\
\hline Sí & 7 & 13 & 3,31 & $0,84-13,2$ & 0,09 \\
\hline No & 7 & 43 & 1,00 & & \\
\hline Hacinamiento ${ }^{d}$ & & & & & \\
\hline Sí & 8 & 29 & 1,24 & $0,33-4,72$ & 0,72 \\
\hline No & 6 & 27 & 1,00 & & \\
\hline Rociar insecticidas habitualmente & & & & & \\
\hline Sí & 3 & 24 & 0,36 & $0,07-1,64$ & 0,14 \\
\hline No & 11 & 32 & 1,00 & & \\
\hline
\end{tabular}

a OR: Razón de posibilidades cruda.

b IC95\%: intervalo de confianza de $95 \%$.

${ }^{\mathrm{c}}$ Nivel de significación $P<0,05$.

${ }^{d}$ Hacinamiento: cuando en una misma habitación duermen 4 personas o más.

El tipo de material de construcción de las viviendas representa también un elemento importante en la dinámica de transmisión de la enfermedad de Chagas, ya que según sus características, el agente transmisor puede permanecer más tiempo y reproducirse dentro de las viviendas (41). En general se observó que el empleo de materiales de construcción de mayor riesgo en el piso aumentaba el riesgo de infestación. Las fisuras en el techo, independientemente del material de construcción empleado, constituyó un factor de riesgo adicional. Según los resultados obtenidos, si se hubieran reparado las fisuras de los techos de las viviendas, se hubiera podido evitar la infección de $73 \%$ de los casos encontrados. En general, se podría haber evitado $81 \%$ de los casos infectados si se hubieran erradicado los triatominos de las viviendas.

No obstante, estas medidas no permiten eliminar totalmente el riesgo de transmisión debido a que esta podría ocurrir en los alrededores del domicilio, en áreas silvestres o mediante otros mecanismos. La transmisión vertical es la tercera causa más frecuente de adquisición de la enfermedad. Según estudios realizados en América del Sur, los hijos de madres positivas tienen 3,9 veces más posibilidades de presentar anticuerpos contra T. cruzi que los hijos de madres negativas $(10,11,17,42,43)$.

Según diversos autores, la aplicación de medidas más estrictas de control de la transmisión en el grupo de niños en edad escolar ha permitido reducir el nivel de la infección con T. cruzi $(14-16,44)$. Los datos de prevalencia de anticuerpos contra $T$. cruzi pueden servir como indicadores del nivel de transmisión activa y para hacer el seguimiento y evaluar la eficacia de los programas de control de vectores $(10,17,41,42)$.

Las comunidades en las que se detecten niños menores de 7 años positivos a anticuerpos contra $T$. cruzi se deben considerar en riesgo de transmisión de la enfermedad de Chagas y en ellas deben implementarse medidas de control y vigilancia (45).

En resumen, los resultados del presente trabajo demuestran que la seroprevalencia de anticuerpos contra T. cruzi en menores de 18 años del estado de Veracruz fue de $0,91 \%$. Se confirmó la transmisión vectorial activa en la zona y que el ver chinches dentro de la casa y la presencia de fisuras en el techo fueron las principales variables asociadas con la seroprevalencia estudiada. La única especie encontrada tanto dentro como en los alrededores de los domicilios fue Triatoma dimidiata; los índices entomológicos más elevados fueron los de infestación y de colonización.

Se debe seguir atentamente la seroprevalencia en este segmento de la po- 
CUADRO 8. Variables asociadas con la positividad a anticuerpos contra Trypanosoma cruzi en menores de 18 años, según los resultados de la regresión logística. Estado de Veracruz, México, marzo de 2001

\begin{tabular}{lrrrr}
\hline \multicolumn{1}{c}{ Variable } & $\beta$ & OR $^{\mathrm{a}}$ & $\mathrm{IC95}^{\mathrm{b}}$ & \multicolumn{1}{c}{$P^{\mathrm{c}}$} \\
\hline Se vieron chinches dentro de la casa & 1,673 & 5,33 & $1,46-19,5$ & 0,011 \\
Fisuras en el techo & 1,306 & 3,69 & $0,96-14,2$ & 0,05 \\
Constante & $-2,892$ & & & \\
\hline
\end{tabular}

a OR: Razón de posibilidades ajustada por el modelo multifactorial.

b IC95\%: intervalo de confianza de $95 \%$.

${ }^{c}$ Nivel de significación $P<0,05$.

FIGURA 2. Índices entomológicos en las jurisdicciones sanitarias donde se detectaron casos positivos a anticuerpos contra Trypanosoma cruzi. Estado de Veracruz, México, marzo de 2001

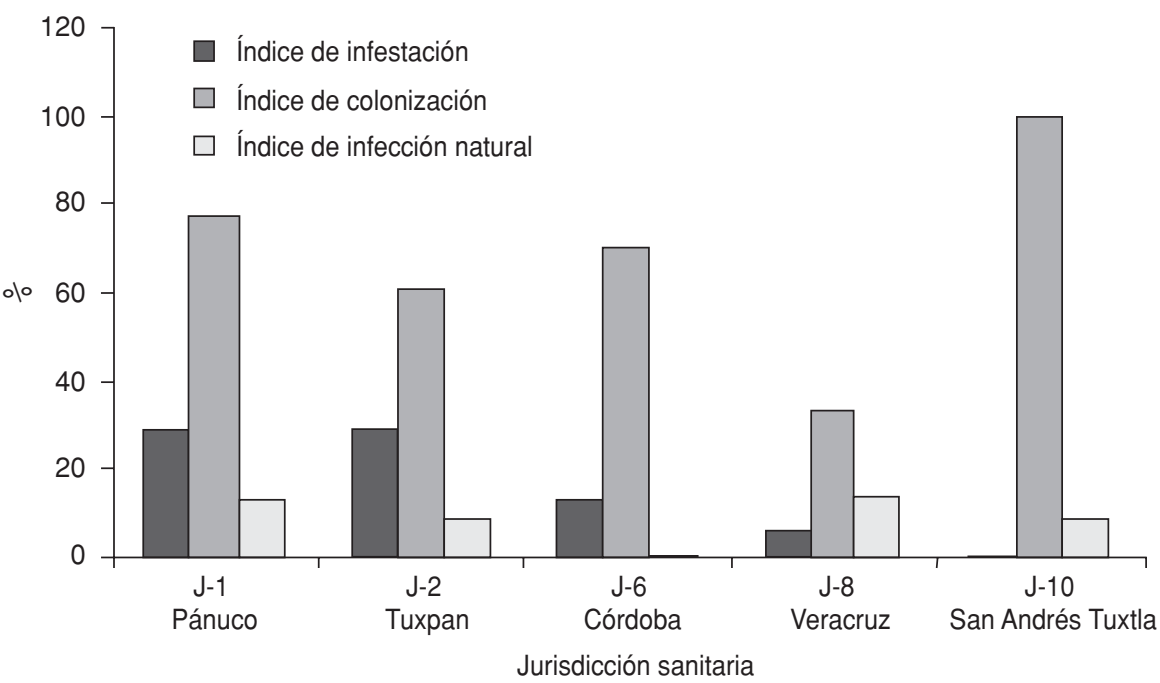

REFERENCIAS

1. Velasco O, Valdespino L, Tapia C. Seroepidemiología de la enfermedad de Chagas en México. Salud Publica Mex. 1992;34:186-96.

2. Biagi F, Tay J, Guzmán C, Fong F. Tetitlán Guerrero: foco endémico de enfermedad de Chagas. Rev Fac Med UNAM (México). 1964; 6:625-31.

3. Quintal R, Zavala J, Rodríguez M. La enfermedad de Chagas en el estado de Yucatán, México. Rev Invest Clin. 1975;27:255-8.

4. Tay J, Salazar-Schettino PM, Velasco CM, De Haro I, García YY, Gutierrez QM. Estudio epidemiológico de la enfermedad de Chagas en el estado de Jalisco, República Mexicana. Salud Publica Mex. 1979:20:145-9.

5. Goldsmith RS, Kagan IG, Zárate R, Reyes G, Cedeño-Ferreira J. Estudios epidemiológicos de la enfermedad de Chagas en Oaxaca, México. Bol Oficina Sanit Panam. 1979;87: 1-19.

6. Goldsmith RS, Ortega M, Zárate R, Zárate L, Beltrán F. Seroepidemiologic surveys for Chagas disease in Chiapas, México. Arch Invest Med. 1983;14:43-50.

7. Salazar Schettino PM, Tay J, Ruiz A, Haro I de, Bucio M, Jiménez J, et al. Seropositividad a Trypanosoma cruzi en cuatro grupos de po- blación del estado de Oaxaca. Rev Salud Publica Mex. 1984;26:589-595.

8. Cortés JM, Velasco O, Labastida M, Melchor A, Duarte N, De Torre R. La enfermedad de Chagas en Santiago Yosotiche, Oaxaca, México. Salud Publica Mex. 1985;2:60-5.

9. Sánchez B. Miocardiopatía crónica e infección por Trypanosoma cruzi en una localidad de Morelos y Tabasco [tesis de maestría]. México, D.F.: Universidad Nacional Autónoma de México; 1988.

10. Salvatella R, Rosa R, González M, Basmadjián Y, Combol A, Benavides U, et al. Seroprevalencia de la infección por Trypanosoma cruzi en escolares de seis a doce años de edad de tres departamentos endémicos de Uruguay. Bol Chil Parasitol. 1999;54:51-6.

11. Meléndez F, Bachler G, Colvin A, Mejías G, Gutiérrez C, Rojas A, et al. Comparación de la infección por Trypanosoma cruzi en niños menores de 10 años de la V Región, Chile. 19821995. Bol Chil Parasitol. 2000;55:27-30.

12. Luluaga I, Lazzari J. Prevalencia de la enfermedad de Chagas en escolares de la ciudad de Buenos Aires. Rev Argent Cardiol. 2002;70: 364-8. blación, considerado como grupo centinela, a fin de evaluar la conveniencia de aplicar medidas más estrictas de control del vector y de vigilancia epidemiológica. Se recomienda mejorar las condiciones de las viviendas - en particular emplear materiales de construcción que no favorezcan la infestación con el vector-, eliminar las fisuras de los techos y erradicar la infestación y colonización del agente transmisor dentro de las viviendas y en sus alrededores. El tratamiento y la vigilancia epidemiológica de los casos detectados en el estado de Veracruz deben formar parte de su Programa de Control de la Enfermedad de Chagas. Se debe informar a la población y fomentar su participación en las acciones de control y vigilancia epidemiológicos.

Agradecimientos. Los autores agradecen a los doctores Edith Rodríguez y Mauro Loyo y al ingeniero Leopoldo Hidalgo, de los Servicios de Salud del estado de Veracruz, México, por su apoyo en el trabajo de campo. El presente estudio recibió apoyo financiero del Programa Especial para Investigación y Entrenamiento en Enfermedades Tropicales de la OMS mediante el proyecto No. 970854.
13. Sessa PA, Pimentel RR, Ferreira AL, Falqueto A Chagas disease seroprevalence among schoolage children in Espiritu Santo State, Brasil, 1999-2000. Cad Saude Publica. 2002;18: 1765-9.

14. Carvalho ME, da Silva RA, Barata JM, Domingos MF, Ciaravolo RM, Zacharias F. Chagas disease in the southern coastal region of Brazil. Rev Saude Publica. 2003;37:49-58.

15. De Andrade AL, Zicker F, Luquetti AO, Oliveira RM, Silva SA, Souza JM, et al. Surveillance of Trypanosoma cruzi transmission by serological screening of schoolchildren. Bull World Health Organ. 1992;70:625-9.

16. Costa FC, Vitor RW, Antunes CM, Carneiro M. Chagas Disease Control Programme in Brazil: a study of the effectiveness of 13 years of intervention. Bull World Health Organ. 1998;76:385-91.

17. Carneiro M, Moreno EC, Antunes CMF. Nested case-control study in a serological survey to evaluate the effectiveness of a Chagas disease control programme in Brazil. Bull World Health Organ. 2001;79:409-14.

18. Organización Panamericana de la Salud, Organización Mundial de la Salud. Tratamiento de la enfermedad de Chagas. Conclusiones de 
una Consulta Técnica. Ginebra: OMS; 1999. (Documento OPS/HCP/HCT/140/99).

19. Hoffmann CC. Nota acerca de un probable transmisor de la tripanosomiasis humana en el estado de Veracruz. Rev Mex Biol. 1928;8:12-8.

20. Lent H, Wygodzinsky P. Revision of the Triatominae (Hemiptera, Reduviidae) and their significance as vectors of Chagas disease. Bull Am Mus Nat Hist. 1979;163:125-520.

21. Tay J, Salazar-Schettino PM, Bucio I, Zárate R. La enfermedad de Chagas en la República Mexicana. Salud Publica Mex. 1980;22:409-50.

22. Zárate L, Zárate R. A checklist of the Triatominae (Hemiptera:Reduviidae) of Mexico. Int J Entomol. 1985;27(1-2):102-27.

23. Salazar-Schettino PM, De Haro I, Urribarren T. Chagas disease in Mexico. Parasitol Today. 1988;4(12):348-52.

24. Vidal V, Ibáñez S, Martínez C. Infección natural de chinches Triatominae con Trypanosoma cruzi asociadas a las viviendas humanas en México. Salud Publica Mex. 2000;42(6):496-503.

25. Guzmán-Bracho C. Epidemiology of Chagas' disease in Mexico: an update. Trends Parasitol. 2001;17(8):372-6.

26. Carcavallo R, Galíndez Girón I, Jurberg J, Lent $\mathrm{H}$. Atlas of Chagas disease vectors in the Americas. Vol. III. Rio de Janeiro: Editora Fiocruz; 1999.

27. Galvão C, Jurberg J, Carcavallo RU, Mena CA, Galíndez I, Curto de Casas SI. Distribuiçao geográfica e dispersao alti-latitudinal de algunos generos e especies de tribo Triatomini Jeannel, 1919 (Hemiptera: Reduviidae:Triatominae). Mem Inst Oswaldo Cruz. 1998,93(1):33-8.

28. Scheaffer RL, Mendenhall WO. Muestreo irrestricto aleatorio. En: Grepe N, ed. Elementos de muestreo. México, D.F.: Grupo Editorial Iberoamérica; 1987.

29. Enfermedad de Chagas y otras parasitosis. Manual de laboratorio. 8. ${ }^{\mathrm{a}}$ ed. Buenos Aires:
Instituto Nacional de Chagas Dr. Mario Fatala Chabén; 1996.

30. Voller A, Draper C, Bidwell D, Bartlett A. Microplate enzyme-linked immunosorbent assay for Chagas' disease. Lancet. 1975;22: 426-8.

31. Bucio M, Cabrera M, Segura E, Zenteno E, Salazar-Schettino PM. Identification of immunodominant antigens in Mexican strains of Trypanosoma cruzi. Immunol Invest. 1999;28: 257-68.

32. Centers for Disease Control and Prevention (CDC), Department of Health, Education and Welfare. A procedural guide to the performance of the indirect fluorescent antibody test for Toxoplasmosis. Atlanta, Georgia: CDC. 1970.

33. Aguilar J. Caracterización biológica e inmunoquímica de cuatro aislados de T. cruzi [tesis de licenciatura]. México, D.F.: Universidad Nacional Autónoma de México; 2004.

34. García de la Torre G. Tripanosomiasis americana en el estado de Morelos [tesis de maestría]. México, D.F.: Universidad Nacional Autónoma de México; 1998.

35. Silveira A, De Rezende D, Correia M. Risk measure of domestic transmission of Chagas disease, through a new entomological indicator. Mem Inst Oswaldo Cruz. 1984;79(Suppl): 113-5.

36. Hosmer DW, Lemeshow S. Applied logistic regression. New York: John Wiles and Sons; 1989.

37. Guzmán C, García C, Floriani I, Guerrero S, Torres M, Ramírez C. Riesgo de transmisión de Trypanosoma cruzi por transfusión de sangre en México. Rev Panam Salud Publica. 1998;4:94-8.

38. Cabrera M, Bucio M, Rojo J, Bonifaz R, Guevara Y, Salazar PM. Detection of antibodies against Trypanosoma cruzi in blood donors in the General Hospital of Mexico City. Rev Patol Trop. 2004;33:71-80.

39. Machado-Coelho GLL, Vitor RWA, Chiari CA, Antunes CMF. Validity of serology for American Tripanosomiasis with eluates from filter paper. Mem Inst Oswaldo Cruz. 1995; 90(1):59-64.

40. Organización Panamericana de la Salud. Manual de procedimientos de control de calidad para los laboratorios de serología de los bancos de sangre. Washington, D.C.: 1994. (Documento $\mathrm{PAHO} / \mathrm{HPC} / \mathrm{HCT} / 94.21$ ).

41. De Andrade Al, Zicker F, De Oliveira RM, Da Silva IG, Silva SA, De Andrade SS, et al. Evaluation of risk factors for house infestation by Triatoma infestans in Brazil. Am J Trop Med Hyg. 1995;53(5):443-7.

42. Bonametti AM, Castelo A, Ramos LR, Dias E, Mutuko P, Da Silveira JL, et al. Seroprevalence of Trypanosoma cruzi infection in students at the seven-fourteen age range, Londrina, PR, Brazil, in 1995. Mem Inst Oswaldo Cruz. 1998;93(6):727-32.

43. Tortora C, Bejarano I, Dipierri J, Alfaro E, García T. Seroepidemiología de la enfermedad de Chagas en escolares de Jujuy. Medicina. 2000; 60:469-73.

44. Organización Panamericana de la Salud. Taller del Cono Sur sobre Enfermedad de Chagas: conceptualización de la vigilancia epidemiológica. Buenos Aires: OPS; 2003. (Documento OPS/DPC/CD/290/04).

45. Guhl F. Programas en la eliminación de la transmisión de la enfermedad de Chagas en Colombia. Medicina. 2000;22:96-104.

Manuscrito recibido el 1 de junio de 2006. Aceptado para publicación, tras revisión, el 6 de julio de 2007.

ABSTRACT Objectives. To determine the prevalence of Trypanasoma cruzi antibodies among the population under 18 years of age in the state of Veracruz, Mexico, and to identify risk factors associated with housing conditions and vector presence and distribution.

Seroprevalence of Trypanosoma cruzi antibodies and associated risk factors among the population under 18 years of age in Veracruz, Mexico

Methods. A cross-sectional, epidemiological study of the population under 18 years of age living in 10 of the 11 health districts of the state of Veracruz, Mexico, from 2000 to2001. Presence of T. cruzi antibodies was determined by hemagglutination inhibition test (HAI) and enzyme-linked immunosorbent assays (ELISA) of serum samples spotted on filter paper, and confirmed by HAI, ELISA, and indirect immunofluorescence assay (IFA) on serum samples. A questionnaire was administered to determined housing conditions, and entomological indices for triatomines were calculated for both the intra- and peridomiciliary areas. The results were analyzed using bifactorial and multifactorial unconditional logistic regression.

Results. Of the 150 individuals presenting an initial reaction, 14 were confirmed positive ( 5 by HAI, ELISA and IFA; 6 by HAI and ELISA, and 3 by ELISA and IFA), for a total prevalence rate of $0.91 \%$ (95\% CI: 0.85-0.94). All of the confirmed-positive cases were from five of 14 health districts, with the Tuxpan district having the highest prevalence rate: $5.2 \%(95 \% \mathrm{CI}: 1.2-9.0)$. Risk factors included having noticed cracks in the house walls and gaps in the roof. The only species of vector captured was Triatoma dimidiata. The entomologic indices for infestation, colonization, and natural infection were: $10.9 \%, 50.0 \%$, and $9.0 \%$, respectively.

Conclusions. Active, vector-borne transmission of T. cruzi was confirmed in the state of Veracruz, Mexico, with an overall antibody seroprevalence rate of $0.19 \%$ among the population under 18 years of age. Seroprevalence rates among this segment of the population, which serves as a sentinel group, should be closely monitored to determine if more stringent methods of vector control and epidemiological surveillance are needed. 